\title{
Hexapods with Plane-Symmetric Self-Motions
}

\author{
Georg Nawratil \\ Institute of Discrete Mathematics and Geometry, Vienna University of Technology, \\ Wiedner Hauptstrasse 8-10/104, 1040 Vienna, Austria; nawratil@geometrie.tuwien.ac.at; \\ Tel.: +43-1-58801-104362; Fax: +43-1-58801-9104362
}

Received: 30 April 2018; Accepted: 5 June 2018; Published: 8 June 2018

check for updates

\begin{abstract}
A hexapod is a parallel manipulator where the platform is linked with the base by six legs, which are anchored via spherical joints. In general, such a mechanical device is rigid for fixed leg lengths, but, under particular conditions, it can perform a so-called self-motion. In this paper, we determine all hexapods possessing self-motions of a special type. The motions under consideration are so-called plane-symmetric ones, which are the straight forward spatial counterpart of planar/spherical symmetric rollings. The full classification of hexapods with plane-symmetric self-motions is achieved by formulating the problem in terms of algebraic geometry by means of Study parameters. It turns out that besides the planar/spherical symmetric rollings with circular paths and two trivial cases (butterfly self-motion and two-dimensional spherical self-motion), only one further solution exists, which is the so-called Duporcq hexapod. This manipulator, which is studied in detail in the last part of the paper, may be of interest for the design of deployable structures due to its kinematotropic behavior and total flat branching singularities.
\end{abstract}

Keywords: hexapod; self-motion; spatial symmetric rolling; plane-symmetric motion; Duporcq manipulator

\section{Introduction}

In planar kinematics, the instantaneous pole $\mathrm{P}$ traces the so-called fixed/moving polode in the fixed/moving system during the constrained motion of a given mechanism. It is well known that this motion can also be generated by the rolling of the moving polode $\phi$ along the fixed polode $\phi_{0}$ without sliding. If the polodes are symmetric with respect to the pole tangent $t$, then the motion is called planar symmetric rolling (cf. Figure 1, left). In 1826, this motion was first (with the exception of the already known symmetric circle rolling yielding the limacons of Pascal) studied by Quetelet [1], who pointed out the following property (cf. [2]): The path $\times$ of a point $\mathrm{X}$ under this special planar motion can be generated by the reflexion of a point $\mathrm{X}_{0}$ of the fixed system on each tangent of $\phi_{0}$. This can also be reformulated as follows: $x$ can be obtained by a central dilation with center $\mathrm{X}_{0}$ and scale factor 2 (i.e., central doubling) of $\mathrm{X}_{0}$ 's pedal-curve $\mathrm{f}$ with respect to $\phi_{0}$. A detailed study of the planar symmetric rolling was done by Bereis [3], Bottema [4] and Tölke (cf. [2] and the references given therein).

The spherical counterpart of this motion is called spherical symmetric rolling and was extensively studied by Tölke in a series of papers, which are summarized and referenced in [2]. The spherical version of the above given characterization also holds true for the spherical symmetric rolling (cf. Figure 1, right). 

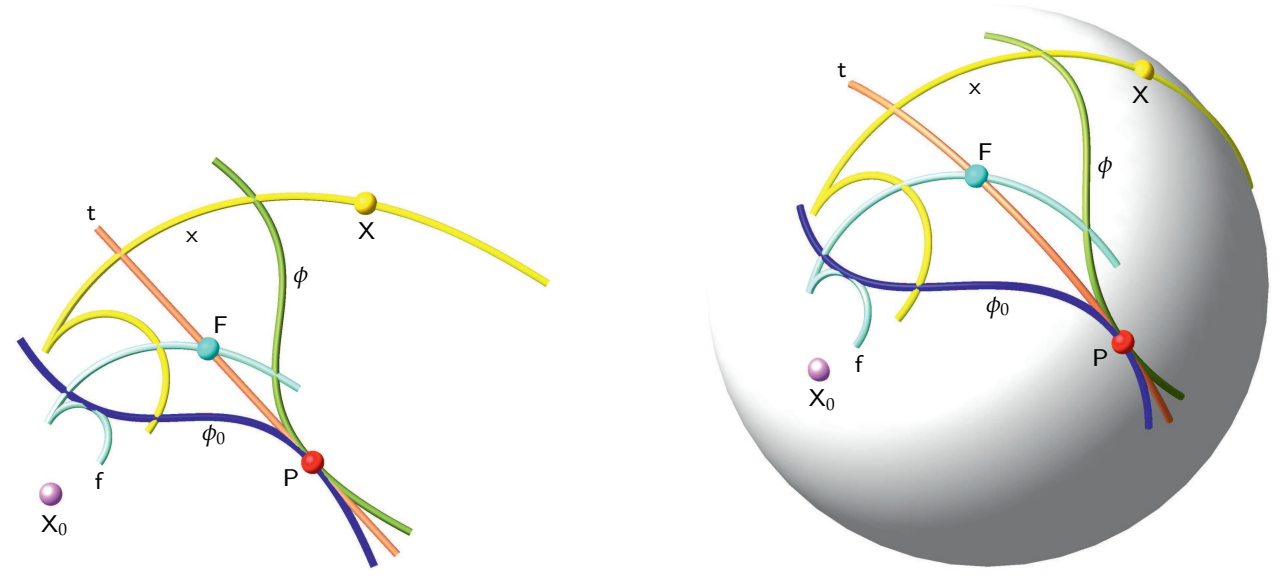

Figure 1. Sketch of the planar symmetric rolling (left) and the spherical symmetric rolling (right). The pedal-point of the fixed point $\mathrm{X}_{0}$ with respect to the pol-tangent $\mathrm{t}$ is denoted by $\mathrm{F}$.

From another perspective, a planar/spherical symmetric rolling can also be generated by reflecting the fixed system in a 1-parametric continuous set of lines/great circles. This point of view is of importance for the spatial generalization of symmetric rollings, which can be done in multiple ways:

1. Darboux noted in [5] (No. 61) a 2-parametric spatial motion, which is generated by the rolling of a moving surface $\Phi$ on an indirect congruent fixed surface $\Phi_{0}$. It also holds that the path-surface of a point $X$ can be generated by the reflexion of a point $X_{0}$ of the fixed system on each tangent-plane of $\Phi_{0}$; for example, the path-surface can be obtained by a central doubling of $\mathrm{X}_{0}$ 's pedal-surface with respect to $\Phi_{0}$ 's tangent-planes.

2. Krames [6] considered the so-called line-symmetric motion as the 1-parametric spatial analogue of the planar/spherical symmetric rolling. These motions are obtained by reflecting the moving system in a 1-parametric continuous set of lines, which form the so-called basic surface $\Gamma$ (cf. Figure 2, left). Krames reasoned this by the fact that the path $x$ of a point $X$ under a line-symmetric motion can be generated by the reflexion of a point $X_{0}$ on each generator $g$ of $\Gamma$; for example, $x$ can be obtained by a central doubling of $X_{0}$ 's pedal-curve $f$ with respect to $\Gamma$ 's rulings. However, it should be pointed out that $\Gamma$ differs from the fixed axode $\Phi_{0}$ (generated by the central tangents of $\Gamma$ ). However, $\Phi_{0}$ and the moving axode $\Phi$ are at each time instant symmetric with respect to the axis $p$ of the instantaneous screw, which is in general not an instantaneous rotation. For further details and references on this motion type, please see [7,8] ( $\$ 7$ of Ch. 4) and [9].

3. It is astonishing that neither Tölke [2] (Section 3.1) nor Krames [6] (p. 394) mentioned the more apparent generalization by reflecting the fixed system in a 1-parametric continuous set of planes. Less attention was paid to these so-called plane-symmetric motions in the literature until now. We summarize the known results in the next section.

Remark 1. Note that the term plane-symmetric motion was also used in [10] (\$3.3) for a superset of the above described motions, which is characterized by the sole property that "the same equation describes the motion and its inverse, but with respect to reference systems that are a reflection of each other". In order to avoid confusions, we point out that we do not mean this superset by using this wording. 

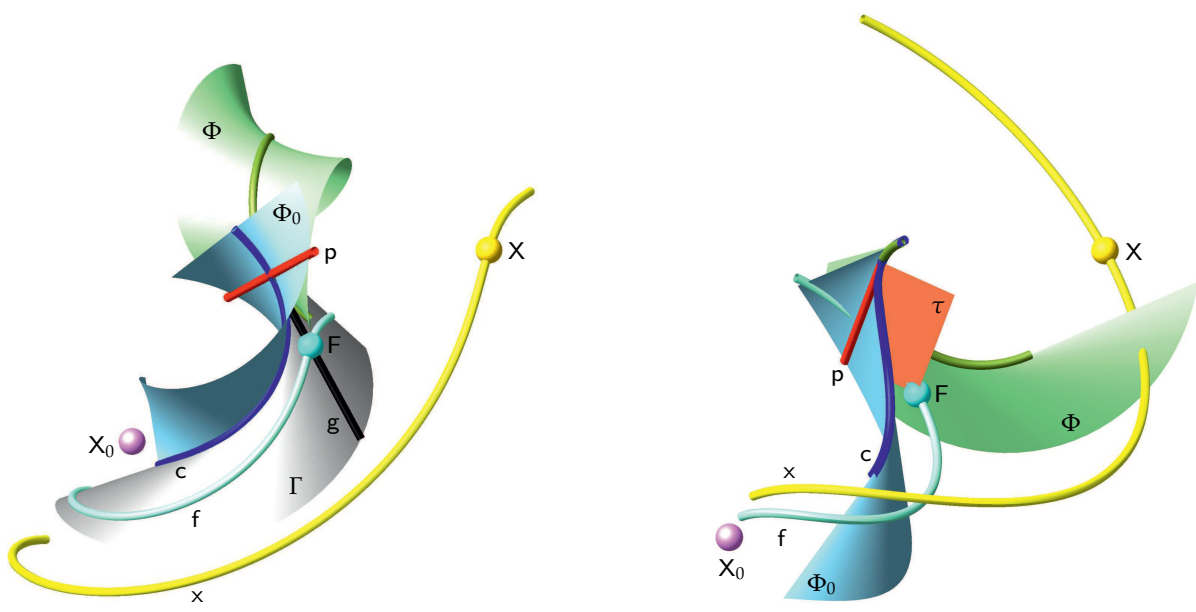

Figure 2. Sketch of the line-symmetric motion (left) and the spatial symmetric rolling (right). For the illustrations, the basic surface $\Gamma$ of the line-symmetric motion and the fixed axode $\Phi_{0}$ of the spatial symmetric rolling have been chosen as tangent-surfaces of a straight cubic circle c. $F$ denotes the pedal-point of the fixed point $X_{0}$ with respect to (left) the generator $g$ of $\Gamma$ and (right) the tangent-plane $\tau$ along the instantaneous axis $p$ of rotation, respectively.

\subsection{Review on Plane-Symmetric Motions}

The basic properties of this motion type are reported in [8] ( $\$ 8$ of Ch. 4). Given is a 1-parametric continuous set of planes $\tau(t)$, where the parameter $t$ can be seen as time. By reflecting the fixed frame $\mathfrak{F}_{0}$ on the plane $\tau(t)$, we obtain the pose $\mathfrak{F}_{0}^{t}$ of the plane-symmetric motion.

Let us consider to infinitesimal neighboring poses $\mathfrak{F}_{0}^{t}$ and $\mathfrak{F}_{0}^{t+\Delta t}$ of the plane-symmetric motion. Now, one can transform $\mathfrak{F}_{0}^{t}$ into $\mathfrak{F}_{0}^{t+\Delta t}$ by a reflexion on $\tau(t)$ followed by a further reflexion on $\tau(t+\Delta t)$. It is well known that this is a pure rotation about the line of intersection of $\tau(t)$ and $\tau(t+\Delta t)$. Moreover, this is exactly a torsal ruling of the developable surface enveloped by the given 1-parametric set of planes. As a consequence, the fixed axode $\Phi_{0}$ is a developable surface (It is well known (e.g., [11] (Thms. 5.1.7 and 6.1.3)) that every developable surface is composed of cylindrical, conical or tangent-surfaces) and the corresponding moving axode $\Phi$ is obtained by reflecting $\Phi_{0}$ in $\Phi_{0}$ 's tangent-plane $\tau$ along the instantaneous axis p of rotation (cf. Figure 2, right). Now, the path $\mathrm{x}$ of a point $X$ under a plane-symmetric motion can be generated by the reflexion of a point $X_{0}$ on each tangent-plane $\tau$ of $\Phi_{0}$; i.e., $\times$ can be obtained by a central doubling of $\mathrm{X}_{0}$ 's pedal-curve $\mathrm{f}$ with respect to $\Phi$ 's tangent-planes.

Due to all these properties, the plane-symmetric motion seems to be the straightforward spatial counterpart of the planar/spherical symmetric rolling. Therefore, we call a plane-symmetric motion also a spatial symmetric rolling.

As far as the author knows, these spatial symmetric rollings are only explicitly mentioned in a practical example by Kunze and Stachel [12], who pointed out that the relative motion of opposite systems of a threefold-symmetric Bricard linkage (e.g., the invertible cube of Schatz) is a plane-symmetric one. Clearly, this also holds for the more general class of plane-symmetric Bricard linkages [13], where the two opposite systems not containing a rotation-axis spanning the plane of symmetry also possess a plane-symmetric relative motion during the overconstrained motion of the closed 6R-chain.

\subsection{Motivation and Outline}

One of the author's main research interests are hexapods with self-motions, i.e., overconstrained parallel manipulators where the platform is linked with the base by six legs, which are anchored via spherical red joints (Due to the spherical joints at the platform and the base, each leg can rotate about its carrier line without changing the pose of the platform. These uncontrolled leg-movements are not 
meant by the term self-motion). All these mechanical devices are solutions to the still unsolved problem posed by the French Academy of Science for the Prix Vaillant of the year 1904, which is also known as Borel-Bricard problem and reads as follows [14]: "Determine and study all displacements of a rigid body in which distinct points of the body move on spherical paths." In order to avoid trivial solutions of the problem, the following assumption should hold for the remainder of the article.

Assumption 1. The platform anchor points $\mathrm{m}_{1}, \ldots, \mathrm{m}_{6}$ of the hexapod as well as the corresponding base anchor points $\mathrm{M}_{1}, \ldots, \mathrm{M}_{6}$ should span in each case at least a plane.

It is well known that so-called architecturally singular hexapods (A hexapod is called architecturally singular if the six legs belong in each relative pose of the platform with respect to the base to a linear line complex) possess self-motions in each pose (over $\mathbb{C}$ ). These special solutions to the Borel-Bricard problem are already well studied (A review on this topic is given in [15] (Section 3.1)). The approaches for the determination of non-architecturally singular hexapods recorded in the literature (Note that we do not claim that the following list of given references is complete), can roughly be divided into the following two groups:

1. Assumptions on the geometry of the platform and base; e.g.,

(a) linear mapping between platform and base [16-22],

(b) symmetry properties of platform and base [20-24],

(c) special topology (e.g., octahedral structure [25]),

or a combination of these assumptions (e.g., [20-22])

2. Assumptions on the self-motion; e.g.,

(a) line-symmetric self-motion [9],

(b) type II Darboux-Mannheim self-motion [26],

(c) Schoenflies self-motion [27],

(d) translational self-motion [28],

(e) self-motion of maximal degree [29],

or more generally characterizations like linear relations between direction cosines [30-33].

Note that these assumptions are done in order to reduce the complexity of the problem, as one has to deal with 30 design parameters ( 24 for the geometry and six leg lengths, whereby the number of 30 can be reduced by one due to the freedom of scaling) and six degrees of freedom.

We want to follow the second approach by assuming that the self-motions are symmetric rollings. Therefore, this paper closes a gap as line-symmetric self-motions and point-symmetric (Point-symmetric motions are obtained by reflecting the fixed system in a 1-parametric continuous set of points and according to [7] (Section 8), these motions are pure translations) self-motions are already well-studied $[9,28]$. In addition, this motion-type seems to be a good candidate for self-motions, due to the following property implied by the symmetry of the motion:

Theorem 1. If a point $\mathrm{A}$ of the moving system traces a spherical curve with center $\mathrm{B}_{0}$ during a plane-symmetric motion, then also the point $\mathrm{B}$ of the moving system has a spherical trajectory about the point $\mathrm{A}_{0}$, where $\mathrm{A}$ and $\mathrm{A}_{0}$ as well as $\mathrm{B}$ and $\mathrm{B}_{0}$, are plane-symmetric points of the moving and fixed frame with respect to the tangent-plane $\tau$ along the instantaneous axis $\mathrm{p}$ of rotation. As a consequence, the set of points with spherical trajectories is indirectly congruent to the set of corresponding sphere centers.

In the remainder of the paper, we call the replacement of the point pair $\left(A, B_{0}\right)$ by $\left(B, A_{0}\right)$ the "symmetric leg-replacement". 
Remark 2. Clearly, the lower dimensional version of Theorem 1 is also true for the planar/spherical symmetric rolling. Moreover, Theorem 1 also holds for point-symmetric motions, if "plane-reflection" is substituted by "point-reflection". A similar result holds for line-symmetric motions; one only has to replace "plane-reflection" by "line-reflection" and "indirectly congruent" by "directly congruent" (see e.g., [9]).

The paper is structured as follows: We start with the discussion of planar/spherical symmetric rolling motions with circular paths in Section 2.1. In Section 2.2, we formulate the problem of determining hexapods with plane-symmetric self-motions in terms of algebraic geometry by means of Study parameters. Based on this description, the problem is solved in Section 3. One of the obtained solutions is the so-called Duporcq hexapod, which is discussed in more detail in Section 4. The paper is closed by a conclusion (cf. Section 5).

\section{Preliminary Considerations and Preparatory Work}

As far as the author knows, no hexapods with plane-symmetric self-motions are reported in the literature so far. From known results in planar/spherical kinematics, which are reviewed in the next subsection, we can immediately construct such hexapods.

\subsection{Planar/Spherical Symmetric Rollings with Circular Paths}

Clearly, a pure rotation is a planar/spherical symmetric rolling where every point of the moving system traces a circle. Besides this trivial case, which we meet again under the notation of a so-called butterfly self-motions (cf. later given Theorem 4), the following planar/spherical symmetric rollings with circular trajectories exist:

- The planar symmetric rolling motions with points running on circular paths are well known due to the study of Bereis [3]. In this case, the polodes are either ellipses or hyperbolas and the focals (two real, two complex) of the moving ellipse/hyperbola are running on circles. They are the Burmester points of this motion. These motion can be realized by the mechanisms illustrated in Figure 3.
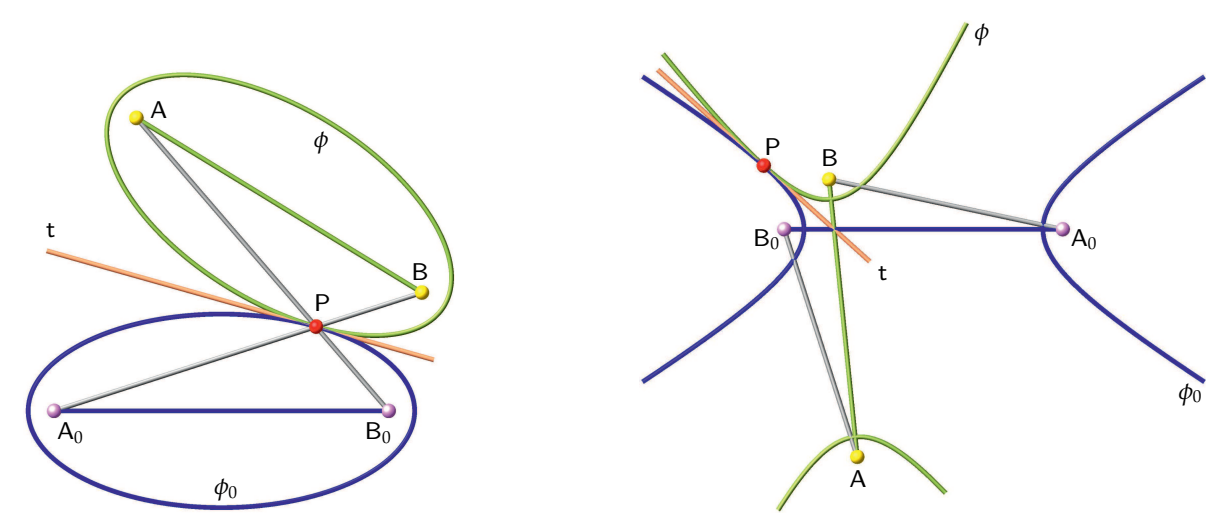

Figure 3. Twin-crank mechanisms with non-counter-rotating cranks (left): In this case, the polodes are ellipses. Twin-crank mechanism with counter-rotating cranks (right): In this case, the polodes are hyperbolas.

- Unfortunately, the considerations of Bereis cannot be generalized straightforward to the sphere (cf. [2] (p. 195)), as in spherical kinematics six Burmester points exist (e.g., [8] (p. 216)). However, we can do the reasoning in a different way. Due to [28] (Theorem 6), one can assume without loss of generality that only two points of a moving body can have spherical trajectories. According to the spherical version of Theorem 1 , a second point is also running on a circle due to the symmetric leg-replacement (With the exceptional case that the first leg is orthogonal to the pole tangent, but 
this will not yield a closed loop; i.e., a spherical parallel manipulator). Thus, we can only end up with a spherical isogram illustrated in Figure 4, which is studied in more detail in [34].
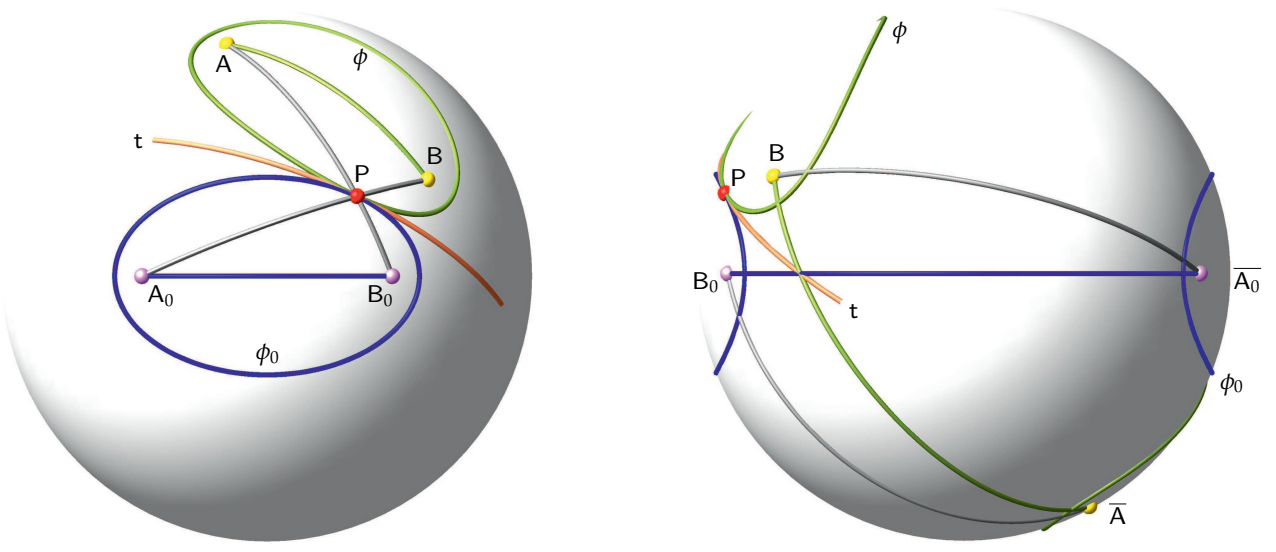

Figure 4. As on the sphere points can be replaced by their antipodes, it can easily be seen that every spherical conic can be interpreted as a spherical ellipse (e.g., [35] (Section 10.1)). The left and the right figure show the same symmetric rolling motion. If we replace $A$ and $A_{0}$ by their antipodal points $\bar{A}$ and $\overline{A_{0}}$, respectively, and look on the sphere from the right side, then we get the figure illustrated on the right-hand side.

From the discussed planar and spherical case, one can easily construct hexapods with plane-symmetric self-motions (see Figure 5).

Remark 3. Note that the hexapods of Figure 5 do not only possess the illustrated plane-symmetric self-motions, but also the already mentioned butterfly self-motions (cf. later given Theorem 4).
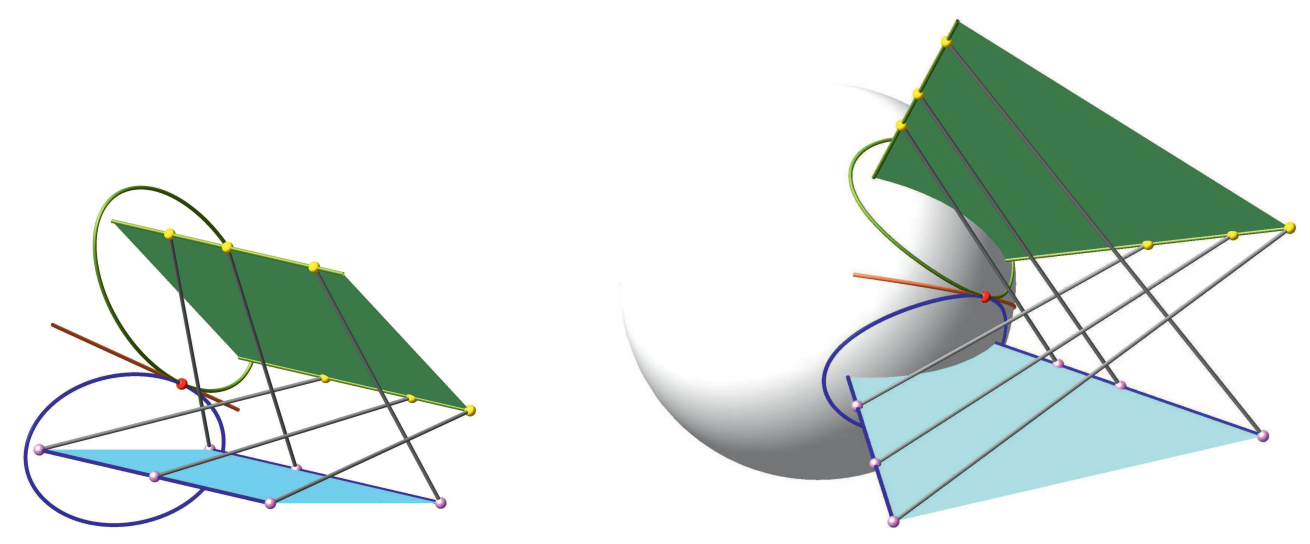

Figure 5. Hexapods with plane-symmetric self-motions, where the platform (green) and the base (blue) are both planar. The axodes of the self-motions are cylinders (left) and cones (right), respectively, but we only illustrated the planar/spherical directrices of these singular quadrics to see better their connection to the planar/spherical symmetric rolling displayed in Figures 3 and 4, respectively. 


\subsection{Mathematical Framework}

For the algebraic formulation of our problem, we want to use Study parameters $\left(e_{0}: e_{1}: e_{2}: e_{3}:\right.$ $\left.f_{0}: f_{1}: f_{2}: f_{3}\right)$, which are nothing else than homogenized dual unit-quaternions $\mathfrak{E}+\varepsilon \mathfrak{F}$ with

$$
\mathfrak{E}=e_{0}+e_{1} \mathbf{i}+e_{2} \mathbf{j}+e_{3} \mathbf{k} \text { and } \mathfrak{F}=f_{0}+f_{1} \mathbf{i}+f_{2} \mathbf{j}+f_{3} \mathbf{k},
$$

where $\mathbf{i}, \mathbf{j}, \mathbf{k}$ are the well-known quaternionic units and $\varepsilon$ the dual unit with the property $\varepsilon^{2}=0$.

Now, all real points of the seven-dimensional Study parameter space $P^{7}$, which are located on the so-called Study quadric $\Psi: \sum_{i=0}^{3} e_{i} f_{i}=0$, correspond to a Euclidean displacement with exception of the three-dimensional subspace $E$ of $\Psi$ given by $e_{0}=e_{1}=e_{2}=e_{3}=0$, as its points cannot fulfill the condition $N \neq 0$ with $N=e_{0}^{2}+e_{1}^{2}+e_{2}^{2}+e_{3}^{2}$. The translation vector $\mathbf{t}:=\left(t_{1}, t_{2}, t_{3}\right)^{T}$ and the rotation matrix $\mathbf{R}:=\left(r_{i j}\right)$ of the corresponding Euclidean displacement $\mathbf{x} \mapsto \mathbf{R} \mathbf{x}+\mathbf{t}$ are given by:

$t_{1}=2\left(e_{0} f_{1}-e_{1} f_{0}+e_{2} f_{3}-e_{3} f_{2}\right), \quad t_{2}=2\left(e_{0} f_{2}-e_{2} f_{0}+e_{3} f_{1}-e_{1} f_{3}\right), \quad t_{3}=2\left(e_{0} f_{3}-e_{3} f_{0}+e_{1} f_{2}-e_{2} f_{1}\right)$,

and

$$
\mathbf{R}=\left(\begin{array}{ccc}
e_{0}^{2}+e_{1}^{2}-e_{2}^{2}-e_{3}^{2} & 2\left(e_{1} e_{2}-e_{0} e_{3}\right) & 2\left(e_{1} e_{3}+e_{0} e_{2}\right) \\
2\left(e_{1} e_{2}+e_{0} e_{3}\right) & e_{0}^{2}-e_{1}^{2}+e_{2}^{2}-e_{3}^{2} & 2\left(e_{2} e_{3}-e_{0} e_{1}\right) \\
2\left(e_{1} e_{3}-e_{0} e_{2}\right) & 2\left(e_{2} e_{3}+e_{0} e_{1}\right) & e_{0}^{2}-e_{1}^{2}-e_{2}^{2}+e_{3}^{2}
\end{array}\right)
$$

if the normalizing condition $N=1$ is fulfilled.

Clearly, the reflection on a plane is an orientation-reversing congruence transformation, which cannot be described directly by the Study parameters. Therefore, we follow the approach of Selig and Husty [7] (Section 8), which is as follows: We start with a reflexion on a fixed plane; say the $x y$-plane of the fixed frame $\mathfrak{F}_{0}$. By this plane-reflection of $\mathfrak{F}_{0}$, we obtain $\overline{\mathfrak{F}}_{0}$. In addition, we apply the reflexion on the plane $\tau(t)$, which finally yields the pose $\overline{\mathfrak{F}}_{0}^{t}$. As the composition of two plane-reflexions is again a direct congruence transformation, we can describe the plane-symmetric motions in this way. If $\tau(t)$ and the $x y$-plane of $\mathfrak{F}_{0}$ are

- not parallel, then the composition is a rotation about the line of intersection,

- $\quad$ parallel, then the composition is a translation orthogonal to these planes.

This yields that the plane-symmetric motions are given by $e_{3}=f_{0}=f_{1}=f_{2}=0$. Moreover, it should be noted that the Study condition is fulfilled identically, thus the set of plane-symmetric motions corresponds to a three-dimensional generator space $P$ of $\Psi$ which intersects $E$ in a line. Based on this description, we analyze the relation between plane-symmetric motions and line-symmetric ones in the next theorem:

Theorem 2. A plane-symmetric motion is also a line-symmetric one if and only if there exists a linear relation $\alpha e_{0}+\beta e_{1}+\gamma e_{2}+\delta f_{3}=0$ with $(\alpha, \beta, \gamma, \delta) \neq(0,0,0,0)$ between the remaining Study parameters.

Proof. For the proof, we need an algebraic characterization of line-symmetric motions in terms of Study parameters. It is well-known that there always exist, a Cartesian frame in the moving system in a way that $e_{0}=f_{0}=0$ holds for a line-symmetric motion. Then, $\left(e_{1}: e_{2}: e_{3}: f_{1}: f_{2}: f_{3}\right)$ are the Plücker coordinates of the generators of the basic surface with respect to the fixed frame.

A change of the moving system can be achieved by a so-called right multiplication; i.e., $(\mathfrak{E}+\varepsilon \mathfrak{F}) \circ(\mathfrak{R}+\varepsilon \mathfrak{S})$ where $\circ$ stands for the quaternionic multiplication. If we denote this product by $\mathfrak{G}+\mathcal{E} \mathfrak{H}$, the corresponding entries $g_{0}$ and $h_{0}$ read as follows (under consideration of $e_{3}=f_{0}=f_{1}=f_{2}=0$ ):

$$
g_{0}:=r_{0} e_{0}-r_{1} e_{1}-r_{2} e_{2}, \quad h_{0}:=s_{0} e_{0}-s_{1} e_{1}-s_{2} e_{2}-r_{3} f_{3} .
$$


If $\delta=0$ holds, then we set $r_{0}=\alpha, r_{1}=-\beta, r_{2}=-\gamma$ and $s_{0}=s_{1}=s_{2}=r_{3}=0$. For $\delta \neq 0$, we set $s_{0}=\alpha, s_{1}=-\beta, s_{2}=-\gamma, r_{3}=-\delta$ and $r_{0}=r_{1}=r_{2}=0$. For both cases, we get $g_{0}=h_{0}=0$, which finishes the sufficiency of the linear relation between $e_{0}, e_{1}, e_{2}, f_{3}$.

Its necessity can also be seen from Equation (2), as without such a linear relation, the condition $g_{0}=h_{0}=0$ can only be fulfilled for $r_{0}=r_{1}=r_{2}=r_{3}=0$, which yields a contradiction as $\Re$ has to differ from the zero-quaternion.

A further important theorem in this context is the following:

Theorem 3. A plane-symmetric motion is also a line-symmetric one if and only if it is a planar motion or a spherical motion.

Proof. If the linear relation equals $f_{3}=\alpha e_{0}+\beta e_{1}+\gamma e_{2}$, then it can easily be checked by direct computations that the point $(\gamma,-\beta,-\alpha)$ is mapped to the point $(\gamma,-\beta, \alpha)$ for all $e_{0}, e_{1}, e_{2}$ fulfilling $N=1$. Therefore, $(\gamma,-\beta, \alpha)$ is the center of the spherical motion.

If the linear relation equals $\alpha e_{0}+\beta e_{1}+\gamma e_{2}=0$, then it can easily be checked by direct computations that the direction $(\gamma,-\beta,-\alpha)$ is mapped to the direction $(\gamma,-\beta, \alpha)$ for all $e_{0}, e_{1}, e_{2}$ fulfilling $N=1$. Therefore, the direction $(\gamma,-\beta, \alpha)$ remains fixed under the motion. Moreover, the translation vector $\left(t_{1}, t_{2}, t_{3}\right)$ is orthogonal to this direction, which already proves that the motion is planar.

These two theorems imply the following statement:

Corollary 1. If we embed the planar and spherical symmetric rollings into SE(3), then they can also be seen as line-symmetric motions.

Therefore, the self-motions of the hexapods illustrated in Figure 5 are plane-symmetric and line-symmetric at the same time. This raises also the question of whether self-motions exist, which are plane-symmetric but not line-symmetric. The answer is given within the next section.

\section{Plane-Symmetric Self-Motions}

The coordinate vector of the base point $\mathrm{M}_{i}$ with respect to the fixed system is given by $\mathbf{M}_{i}=\left(A_{i}, B_{i}, C_{i}\right)^{T}$. The position of the corresponding platform anchor point $\mathbf{m}_{i}(t)$ is obtained by reflecting a point $\mathrm{m}_{i, 0}$ with fixed coordinates $\mathbf{m}_{i}=\left(a_{i}, b_{i}, c_{i}\right)^{T}$ in a 1-parametric continuous set of planes $\tau(t)$. Instead of these reflexions, we use direct isometries based on the Study representation described in Section 2.2 (i.e., $e_{3}=f_{0}=f_{1}=f_{2}=0$ ). Therefore, the locus of the corresponding platform anchor point $\mathbf{m}_{i}$ with respect to the fixed frame can be parametrized as $\mathbf{R} \overline{\mathbf{m}}_{i}+\mathbf{t}$ with $\overline{\mathbf{m}}_{i}=\left(a_{i}, b_{i},-c_{i}\right)^{T}$.

The condition that the point $\mathrm{m}_{i}$ is located on a sphere centered in $\mathrm{M}_{i}$ with radius $d_{i}$ is a quadratic homogeneous equation in the Study parameters according to Husty [36]. For our setup, this so-called sphere condition $\Lambda_{i}$ has the following form:

$$
\begin{aligned}
\Lambda_{i}: & \left(a_{i}^{2}+b_{i}^{2}+c_{i}^{2}+A_{i}^{2}+B_{i}^{2}+C_{i}^{2}-d_{i}^{2}\right) N-4\left(c_{i}+C_{i}\right) e_{0} f_{3}+4\left(b_{i}+B_{i}\right) e_{1} f_{3}-4\left(a_{i}+A_{i}\right) e_{2} f_{3} \\
& -2\left(a_{i} A_{i}+b_{i} B_{i}-c_{i} C_{i}\right) e_{0}^{2}-2\left(a_{i} A_{i}-b_{i} B_{i}+c_{i} C_{i}\right) e_{1}^{2}+2\left(a_{i} A_{i}-b_{i} B_{i}-c_{i} C_{i}\right) e_{2}^{2} \\
& -4\left(c_{i} B_{i}+b_{i} C_{i}\right) e_{0} e_{1}+4\left(c_{i} A_{i}+a_{i} C_{i}\right) e_{0} e_{2}-4\left(b_{i} A_{i}+a_{i} B_{i}\right) e_{1} e_{2}+4 f_{3}^{2}=0 .
\end{aligned}
$$

It corresponds to a quadric in the three-dimensional projective space $P^{3}$ with homogenous coordinates $\left(e_{0}: e_{1}: e_{2}: f_{3}\right)$. The symmetric leg-replacement (cf. Theorem 1$)$ can also easily be seen within this formula, as it is invariant under the following permutations: $A_{i} \leftrightarrow a_{i}, B_{i} \leftrightarrow b_{i}, C_{i} \leftrightarrow c_{i}$. Due to this symmetry, we only have to find spatial rolling motions where three points have a spherical trajectory. This means that the corresponding three quardrics $\Lambda_{1}, \Lambda_{2}$ and $\Lambda_{3}$ of $P^{3}$ have to have a curve in common, which can be a 
1. straight line,

2. conic section,

3. cubic curve,

4. quartic curve.

In the following subsections these cases are discussed separately.

\subsection{Intersection Curve Is a Straight Line}

It is well-known that straight lines in the Study quadric correspond with either rotations about a line or straight translations. As the second option is not possible due to the sphere condition, we are only left with the rotation case. In the first step, we ask under which conditions two quadrics $\Lambda_{1}$ and $\Lambda_{2}$ have a straight line in common.

- General Case: Let us assume that $\mathrm{M}_{1} \neq \mathrm{M}_{2}$ and $\mathrm{m}_{1}(t) \neq \mathrm{m}_{2}(t)$ hold. Clearly, the straight line in $P^{3}$ has to correspond with a rotation about the line $G$ spanned by $M_{1}$ and $M_{2}$. Therefore, the line $g(t)$ spanned by $\mathrm{m}_{1}(t)$ and $\mathrm{m}_{2}(t)$ generates either a hyperboloid, cone or cylinder of revolution with axis $\mathrm{G}$. Moreover, all these poses of the platform points have to be obtained by plane-reflexions of the points $m_{1,0}$ and $m_{2,0}$, respectively. This already implies that the 1-parametric set of planes $\tau(t)$ has to be a pencil of planes with axis G. Therefore, the leg lengths $d_{1}$ and $d_{2}$ are given by

$$
d_{i}=\operatorname{dist}\left(\mathrm{M}_{i}, \mathrm{~m}_{i, 0}\right)=\sqrt{\left(A_{i}-a_{i}\right)^{2}+\left(B_{i}-b_{i}\right)^{2}+\left(C_{i}-c_{i}\right)^{2}},
$$

which is already the necessary and sufficient condition for the two quadrics $\Lambda_{1}$ and $\Lambda_{2}$ to have a straight line in common.

- Special Case: As the case $\mathrm{M}_{1}=\mathrm{M}_{2}$ and $\mathrm{m}_{1}(t)=\mathrm{m}_{2}(t)$ cannot arise (legs are identical), we only have to discuss one further case due to the symmetric leg-replacement. Without loss of generality, we can assume $M_{1} \neq M_{2}$ and $m_{1}(t)=m_{2}(t)$. Now, $m_{1}(t)=m_{2}(t)$ has to trace a circle about the line $G$, which in fact implies the same condition given in Equation (4) for $i=1,2$.

Under consideration of the notation that $\left(\mathrm{M}_{i}, \mathrm{~m}_{i}\right)$ and $\left(\mathrm{M}_{i+3}, \mathrm{~m}_{i+3}\right)$ are coupled by the symmetric leg-replacement (for $i=1,2,3$ ), we can immediately formulate the following theorem.

Theorem 4. Up to symmetric leg-replacements, the three quadrics $\Lambda_{1}, \Lambda_{2}$ and $\Lambda_{3}$ have a line in common if and only if Equation (4) holds for $i=1,2,3$ and $\mathrm{M}_{1}, \mathrm{M}_{2}, \mathrm{M}_{3}$ are collinear. The corresponding self-motion of the hexapod is a butterfly self-motion about the line spanned by $\mathrm{M}_{1}, \mathrm{M}_{2}, \mathrm{M}_{3}$, where $\mathrm{M}_{i}=\mathrm{m}_{i+3}$ holds for $i=1,2,3$.

As these butterfly self-motions (cf. Figure 6, left) are trivial, they are not of further interest.

\subsection{Intersection Curve Is a Conic}

As the conic is a planar curve, there has to exist a linear relation between the homogenous coordinates $\left(e_{0}: e_{1}: e_{2}: f_{3}\right)$ of $P^{3}$. Therefore, we can apply the Theorems 2 and 3 , which imply that we can only end up with planar/spherical symmetric rollings already discussed in Section 2.1.

\subsection{Intersection Curve Is Cubic}

A necessary condition that $\Lambda_{1}, \Lambda_{2}$ and $\Lambda_{3}$ have a cubic curve in common is that the intersection of two quadrics split up into a line and this cubic. Therefore, condition Equation (4) has to hold. It can easily be checked that $\Lambda_{i}$ splits up into two planes:

$$
\Lambda_{i}: \quad 4\left(C_{i} e_{0}-B_{i} e_{1}+A_{i} e_{2}-f_{0}\right)\left(c_{i} e_{0}-b_{i} e_{1}+a_{i} e_{2}-f_{0}\right)=0
$$




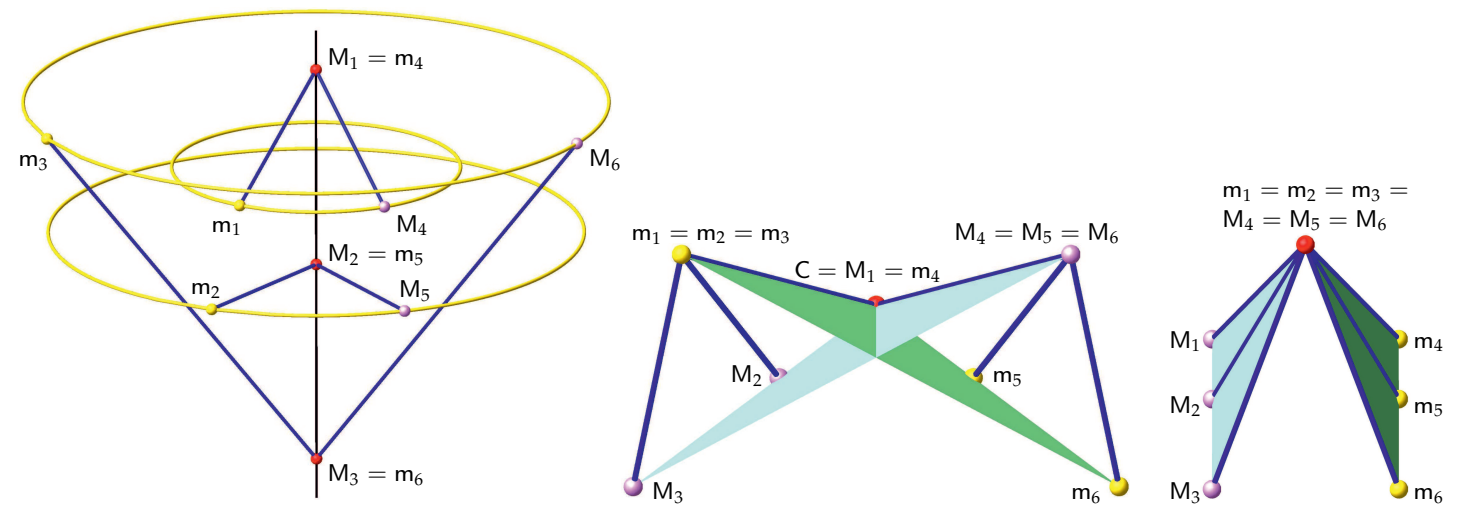

Figure 6. In all three illustrations, the plane of symmetry is always a vertical projecting plane; left: butterfly self-motion of a hexapod. Note that not necessarily the three legs obtained by the symmetric leg-replacements have to be added, but any legs where neither $M_{i}$ or $m_{i}(t)$ is collinear with $\mathrm{M}_{1}, \mathrm{M}_{2}, \mathrm{M}_{3}$ for $i=4,5,6$; center: situation after performing the $\Delta$-transform; right: two-dimensional spherical self-motion.

under consideration of Equation (4). Therefore, the cubic has to split up into three lines, which all correspond to plane-symmetric butterfly self-motions already described in Theorem 4 . As a consequence, no further discussion of this case is necessary.

\subsection{Intersection Curve Is Quartic}

We start with the following lemma, which helps to exclude the discussion of special cases arising.

Lemma 1. If $\mathrm{M}_{1}, \mathrm{M}_{2}, \mathrm{M}_{3}$ are collinear and $\mathrm{m}_{1}=\mathrm{m}_{2}=\mathrm{m}_{3}$ holds (under consideration of symmetric leg-replacements), then the hexapod can only have the following plane-symmetric self-motions:

1. butterfly self-motion,

2. two-dimensional spherical self-motion,

3. planar/spherical symmetric rollings of Section 2.1.

Proof. If the carrier line $G$ of $M_{1}, M_{2}, M_{3}$ is always identical with the reflected carrier line $g$ of $m_{4}, m_{5}, m_{6}$, then it is clear that the motion can only be a butterfly motion (cf. Figure 6, left).

Moreover, it is trivial, that the motion can only be a planar one if $\mathrm{G}$ is always parallel to $\mathrm{g}(\Rightarrow$ planar symmetric rolling of Section 2.1).

Now, we discuss the remaining case that, during the plane-symmetric self-motion, one configuration exists, where $\mathrm{G}$ and $\mathrm{g}$ intersect in one point $\mathrm{C}$. As the first three legs are always in a pencil of lines, one can make a so-called $\Delta$-transform [37] (without changing the self-motion) such that $\mathrm{M}_{1}=\mathrm{C}$ holds. This results in the following relations (cf. Figure 6, center):

$$
\overline{\mathrm{M}_{1} \mathrm{M}_{4}}=\overline{\mathrm{m}_{4} \mathrm{M}_{4}} \text { and } \overline{\mathrm{m}_{1} \mathrm{~m}_{4}}=\overline{\mathrm{M}_{1} \mathrm{~m}_{4}} .
$$

Under consideration of the plane-symmetric setup, these conditions can only be fulfilled if

- $\mathrm{M}_{1}=\mathrm{m}_{4}$ holds, which yields the spherical symmetric rolling (with center $\mathrm{M}_{1}=\mathrm{m}_{4}$ ) of Section 2.1,

- $\mathrm{M}_{4}=\mathrm{m}_{1}$ holds, which implies a two-dimensional spherical self-motion (with center $\mathrm{M}_{4}=\mathrm{m}_{1}$; cf. Figure 6, right).

This finishes the proof. 
Remark 4. Note that, for the two-dimensional spherical self-motion, the collinearity condition of $\mathrm{M}_{1}, \mathrm{M}_{2}, \mathrm{M}_{3}$ is not necessary. For the leg lengths of Equation (4) and $\mathrm{m}_{1}=\mathrm{m}_{2}=\mathrm{m}_{3}$, the three quadrics $\Lambda_{1}, \Lambda_{2}$ and $\Lambda_{3}$ have already a plane in common due to Equation (5).

If $\Lambda_{1}, \Lambda_{2}$ and $\Lambda_{3}$ have a quadric curve in common, they are contained within a pencil of quadrics, which is already spanned by two of them. Therefore, we make the following ansatz:

$$
\Sigma: \quad \lambda_{1} \Lambda_{1}+\lambda_{2} \Lambda_{2}+\Lambda_{3}=0 \text { with } \quad \lambda_{1} \lambda_{2} \neq 0 .
$$

In order to simplify the resulting direct computations, we can select the fixed frame in a clever way based on the following lemma:

Lemma 2. By applying symmetric leg-replacements, we can assume that $\mathrm{M}_{1}, \mathrm{M}_{2}, \mathrm{M}_{3}$ span a plane (under consideration of Assumption 1).

Proof. If $M_{1}, M_{2}, M_{3}$ are collinear (span the line $G$ ), we apply the symmetric leg-replacement to the $i$ th leg for $i \in\{1,2,3\}$. Due to Assumption 1, at least one of the $M_{i+3}$ are not located on $G$, thus, after a renumeration of anchor points, the lemma holds.

Due to Lemma 2, we can assume without loss of generality that the origin of the fixed frame equals $M_{i}$, that $M_{j}$ is located on the positive $x$-axis of the fixed frame and that $M_{k}$ is located in the $x y$-plane of the fixed frame for pairwise distinct $i, j, k \in\{1,2,3\}$. As $M_{1}, M_{2}, M_{3}$ is a triangle there always exist at least four (This number results from the fact that each triangle has at least two acute angles, whose two vertices can be used as $\mathrm{M}_{i}$ ) choices for $i, j, k$ in a way that $\mathrm{M}_{k}$ is located in the 1 st quadrant of the xy-plane. After a may necessary renumeration, we can assume:

$$
\mathbf{M}_{1}=(0,0,0)^{T}, \quad \mathbf{M}_{2}=\left(A_{2}, 0,0\right)^{T}, \quad \mathbf{M}_{3}=\left(A_{3}, B_{3}, 0\right)^{T},
$$

with $A_{2}>0, A_{3}>0$ and $B_{3}>0$. Moreover, by selecting the unit-length in a suitable way, we can achieve $A_{2}=1$.

Based on this choice of the fixed frame, we inspect the coefficients of the linear combination $\Sigma$ given in Equation (7) with respect to the Study parameters. We denote the coefficient of $e_{0}^{i} e_{1}^{j} e_{2}^{k} f_{3}^{l}$ by $\Sigma_{i j k l}$. From $\Sigma_{1100}=-4 c_{3} B_{3}$, we get $c_{3}=0$. Moreover, we can compute $d_{3}^{2}$ from $\Sigma_{2000}$. Then, $\Sigma_{0200}$ equals $4 b_{3} B_{3}$, which implies $b_{3}=0$. From $\Sigma_{1100}=4 \lambda_{2} c_{2}$, we get $c_{2}=0$. Now, $\Sigma_{1001}=4 \lambda_{1} c_{1}$ yields $c_{1}=0$. Then, we express $A_{3}$ and $B_{3}$ from $\Sigma_{0101}$ and $\Sigma_{0011}$, which results in

$$
A_{3}=-a_{3}-\lambda_{1} a_{1}-\lambda_{2} a_{2}-\lambda_{2}, \quad B_{3}=-\lambda_{1} b_{1}-\lambda_{2} b_{2}
$$

Moreover, we can set $\lambda_{1}=-1-\lambda_{2}$ due to $\Sigma_{0002}$. Then, we are only left with the following two conditions arising from $\Sigma_{0110}$ and $\Sigma_{0020}$, respectively:

$$
-a_{3} b_{1}-a_{3} b_{1} \lambda_{2}+a_{3} \lambda_{2} b_{2}-\lambda_{2} b_{2}=0, \quad-a_{3}^{2}+a_{3} a_{1}+a_{3} a_{1} \lambda_{2}-a_{3} \lambda_{2} a_{2}-a_{3} \lambda_{2}+\lambda_{2} a_{2}=0 .
$$

Eliminating $\lambda_{2}$ out of these equations by resultant method yields:

$$
a_{3}\left(a_{3}-1\right)\left(a_{3} b_{1}-a_{3} b_{2}-b_{1} a_{2}+a_{1} b_{2}\right) .
$$

Therefore, we distinguish the following cases:

1. For $a_{3}=0$, Equation (10) imply $\lambda_{2} a_{2}=0$ and $\lambda_{2} b_{2}=0$, respectively. $a_{2}=b_{2}=0$ imply the conditions of Lemma 1.

2. For $a_{3}=1$, Equation (10) imply $\lambda_{2}=-1$. Then, the second and third leg are identical under consideration of symmetric leg-replacement. 
3. For $a_{3} b_{1}-a_{3} b_{2}-b_{1} a_{2}+a_{1} b_{2}=0$, we have to distinguish two cases:

(a) $b_{1}=b_{2}$ : now, the condition simplifies to $b_{1}\left(a_{1}-a_{2}\right)=0$. As $b_{1}=0$ implies $B_{3}=0$ a contradiction, we set $a_{1}=a_{2}$. Then, Equation (10) imply $\lambda_{2}=-a_{3}$, which results in the conditions of Lemma 1.

(b) $\quad b_{1} \neq b_{2}$ : Under this assumption, we can solve this equation for $a_{3}$. A further two cases have to be distinguished:

i. $b_{2} a_{1}-b_{1} a_{2}-b_{2}=0$ : If one solves this equation for $a_{i}$, then Equation (10) implies $b_{j}=0$ for distinct $i, j \in\{1,2\}$. In both cases, we end up with the conditions of Lemma 1.

ii. $\quad b_{2} a_{1}-b_{1} a_{2}-b_{2} \neq 0$ : Under this assumption, we can solve the condition implied by Equation (10) for $\lambda_{2}$, which yields:

$$
\lambda_{2}=\frac{\left(b_{1} a_{2}-b_{2} a_{1}\right) b_{1}}{\left(b_{1}-b_{2}\right)\left(b_{2} a_{1}-b_{1} a_{2}-b_{2}\right)} .
$$

It can easily be checked that the obtained solution corresponds to the hexapod's platform and base illustrated in Figure 7, which are also known as Duporcq's complete quadrilaterals [38]. In the remainder of the paper, this interesting solution, which is discussed/studied in more detail in the next section, is called Duporcq hexapod. Based on this notation, we can formulate the following theorem.

Theorem 5. Besides the trivial cases mentioned in Lemma 1, the quadrics $\Lambda_{1}, \Lambda_{2}$ and $\Lambda_{3}$ belong to a pencil if and only if they correspond to sphere conditions of three legs of a Duporcq hexapod (which are not identical under symmetric leg-replacements).
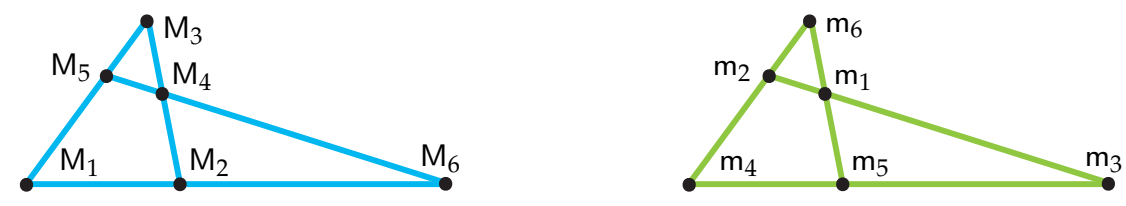

Figure 7. Illustration of Duporcq's complete quadrilaterals: The base (left) is congruent with the platform (right).

\section{Duporcq Hexapod}

Due to the results obtained in Section 3 and Theorems 2 and 3, we can conclude that only the Duporcq hexapod of Theorem 5 possesses plane-symmetric self-motions, which are neither planar nor spherical motions. Therefore, we discuss this hexapod in more detail in this section.

In [38], Duporcq describes the following remarkable motion: Let $\mathrm{M}_{1}, \ldots, \mathrm{M}_{6}$ and $\mathrm{m}_{1}, \ldots, \mathrm{m}_{6}$ be the vertices of two complete quadrilaterals, which are congruent. Moreover, the vertices are labeled in a way that $\mathrm{m}_{i}$ is the opposite vertex of $\mathrm{M}_{i}$ for $i \in\{1, \ldots, 6\}$ (cf. Figure 7). Then, there exists a 2-parametric line-symmetric motion where each $\mathrm{m}_{i}$ is running on spheres centered in $\mathrm{M}_{i}$.

It is well known [39] (Section 1) that this is an architecturally singular hexapod and that one can remove any leg without changing the direct kinematics of the mechanism. The resulting pentapod is called Duporcq pentapod and its line-symmetric self-motions were also studied in [39]. For the coordinatisation of the platform points and base points used in Section 3.4, the 2-parametric line-symmetric self-motion fulfills $e_{0}=f_{0}=0$ (cf. [39] (Section 4)). 
Remark 5. Note that the theoretic results of Section 4 are visualized on the basis of the following example:

$$
a_{1}=\frac{3}{2}, \quad a_{2}=b_{1}=3, \quad b_{2}=\frac{9}{4}, \quad d_{1}^{2}=\frac{17}{2}, \quad d_{2}^{2}=\frac{33}{2} .
$$

This input data implies

$$
a_{3}=\frac{15}{2}, \quad A_{3}=\frac{8}{7}, \quad B_{3}=\frac{6}{7}, \quad d_{3}^{2}=\frac{13231}{196},
$$

with respect to the coordinatisation of the platform points and base points used in Section 3.4.

\subsection{Plane-Symmetric Self-Motions of the Duporcq Hexapod}

First of all, it should be pointed out that the plane-symmetric self-motions of the Duporcq manipulator were not known until now. They can be computed as follows: We express $f_{3}$ from the condition $\Lambda_{2}-\Lambda_{1}$ (which is linear in $f_{3}$ ). Plugging the resulting expression into $\Lambda_{1}$ implies a homogenous quartic equation $\mathrm{Y}$ in $e_{0}, e_{1}, e_{2}$, which already represents the plane-symmetric self-motion (cf. Figure 8, left).

In the following, we are interested in the transition poses between this one-dimensional plane-symmetric self-motion and the above-mentioned two-dimensional line-symmetric one. Therefore, we only have to intersect the quartic curve $\mathrm{Y}$ with $e_{0}=0$, which yields four of these so-called branching singularities [40]. These four transition poses are totally flat configurations of the Duporcq hexapod (cf. Table 1, Figure 8, red left and Figure 9).

Remark 6. Note that a further prominent example of a hexapod, which possesses flat poses during its self-motion, is Bricard's flexible octahedron of type 3 (cf. [41]).

Moreover, it should be mentioned that the Duporcq hexapod is a kinematotropic mechanism (according to the notation of Wohlhart [42]). To the best of the author's knowledge, only one further hexapod with this property is known so far, which is the so-called Wren platform (see [42] (Section 3) and [21] (Section 2.2)).
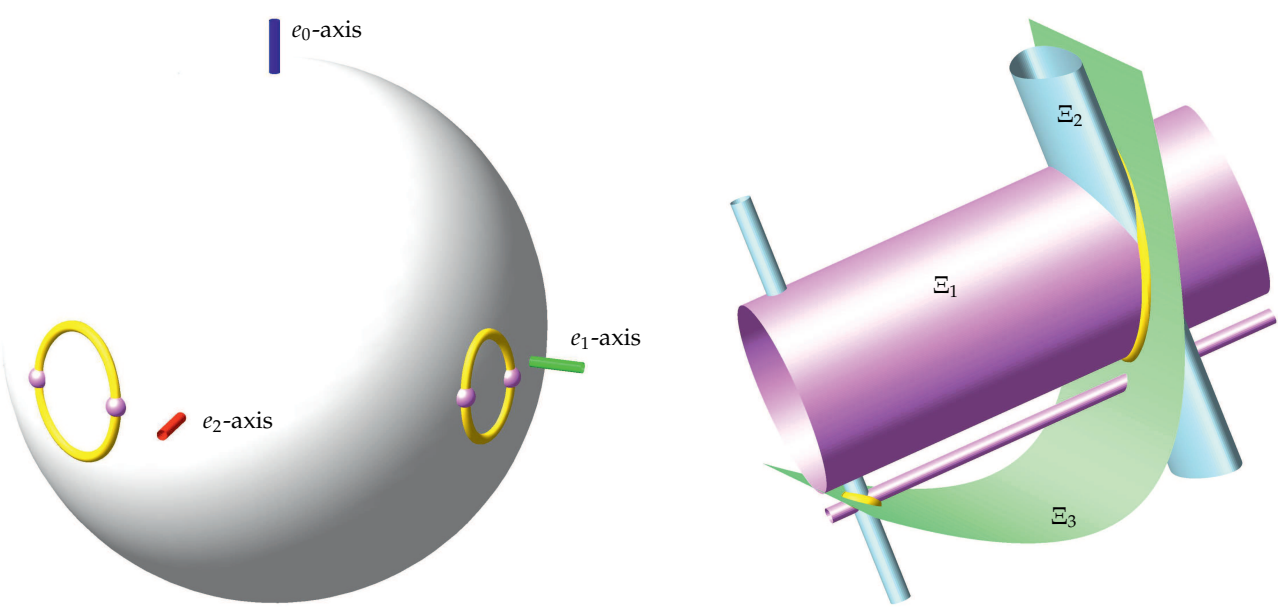

Figure 8. (left:) the quartic $\mathrm{Y}$ is displayed under consideration of the normalization condition $N=1$. For the example at hand, it consists of two components (as antipodal points yield the same displacement). Intersection points of the displayed spherical curve with the equator plane yield the branching singularities between plane-symmetric and line-symmetric self-motions. They are numbered from left to right by 1 to 4 . (right): visualization of the surfaces $\Xi_{i}$ under the assumption that $u_{0}=0$ corresponds to the ideal plane. The surface $\Xi_{i}$ is a cylinder in direction of the $u_{i}$-axis (for $i=1,2,3$ ). 
Table 1. The Study parameters of the four flat transition poses illustrated in Figure 9. As they result as roots of a polynomial of degree 4 , they can be computed explicitly, but, in order to avoid too long expressions, they are displayed numerically.

\begin{tabular}{cccc}
\hline Flat Pose & $\boldsymbol{e}_{\mathbf{1}}$ & $\boldsymbol{e}_{\mathbf{2}}$ & $\boldsymbol{f}_{\mathbf{3}}$ \\
\hline 1 & -0.63171148011492395006 & 0.77520358996267041460 & 0.24434973773984142590 \\
2 & -0.26236530678800600560 & 0.96496862425367773706 & 0.36840718493416854565 \\
3 & 0.89932040897259076870 & 0.43729029489044469464 & -1.6168042368274940498 \\
4 & 0.98317707611585865513 & 0.18265496708349071532 & -2.3876030965525136289 \\
\hline
\end{tabular}
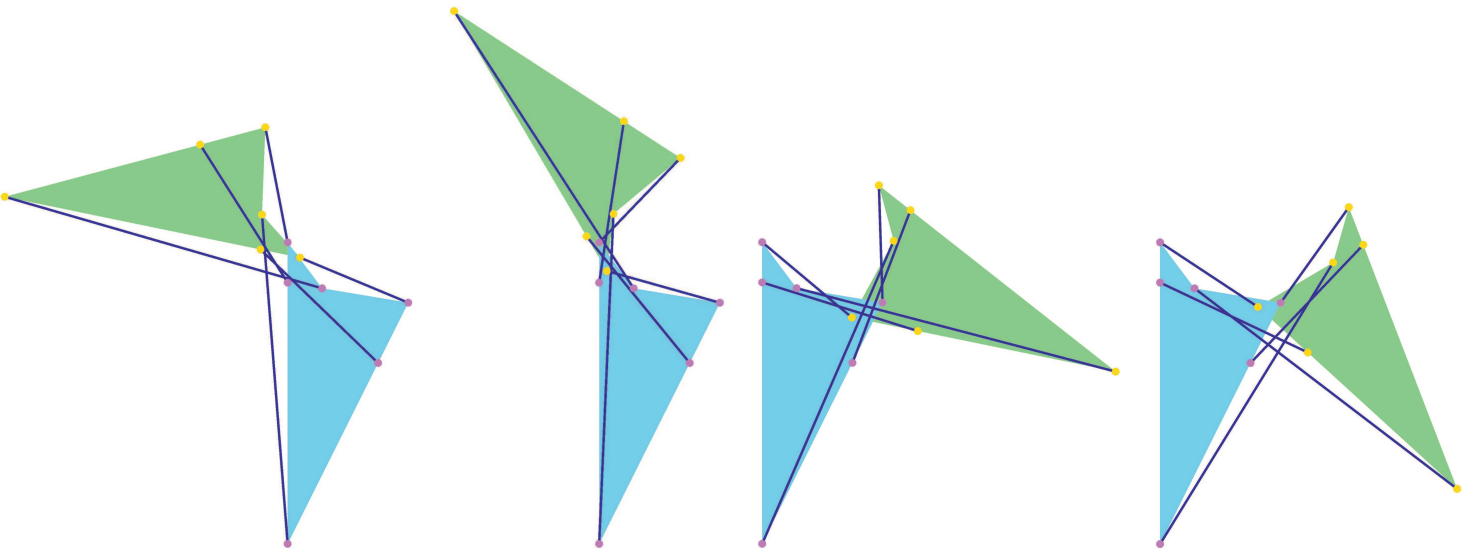

Figure 9. The four flat transition poses numbered from left to right by 1 to 4 .

For the example at hand, the fixed axode can be described in the dual representation (If $a x+b y+c z+d=0$ is the equation of the plane of symmetry, then its dual representation is given by the homogenous quadruplet $\left(u_{0}: u_{1}: u_{2}: u_{3}\right)=(d: a: b: c)$ according to [11] (Section 6.2)) as the intersection of the following three surfaces displayed in Figure 8 , right:

$$
\begin{array}{ll}
\Xi_{1}: \quad & 178596 u_{3}^{2} u_{2}^{2}+45924 u_{0}^{4}+69696 u_{3}^{4}+464508 u_{2}^{3} u_{0}+293436 u_{2} u_{3}^{2} u_{0} \\
& +573049 u_{2}^{2} u_{0}^{2}+124921 u_{3}^{2} u_{0}^{2}+108900 u_{2}^{4}+276336 u_{2} u_{0}^{3}=0, \\
\Xi_{2}: \quad & 108900 u_{1}^{4}+592944 u_{1}^{3} u_{0}+39204 u_{3}^{2} u_{1}^{2}+688345 u_{1}^{2} u_{0}^{2}+361152 u_{3}^{2} u_{1} u_{0} \\
& -300932 u_{1} u_{0}^{3}+18724 u_{0}^{4}+831744 u_{3}^{2} u_{0}^{2}=0, \\
\Xi_{3}: \quad 264 u_{1}^{2}+709 u_{1} u_{0}+198 u_{2} u_{1}+912 u_{2} u_{0}+326 u_{0}^{2}=0 .
\end{array}
$$

Based on these surfaces, it can be checked (e.g., by computing the Hilbert-polynomial) that the fixed axoide corresponds to an algebraic curve of degree 4 in the dual representation. This curve can easily be parametrized as follows (two branches):

$$
u_{0}=1, \quad u_{2}=-\frac{264 u_{1}^{2}+709 u_{1}+326}{6\left(33 u_{1}+152\right)}, \quad u_{3}= \pm \frac{\sqrt{w}}{6\left(33 u_{1}+152\right)},
$$

with $w=300932 u_{1}-18724-688345 u_{1}^{2}-592944 u_{1}^{3}-108900 u_{1}^{4}$. Moreover it can be seen (cf. Figure 8 , right) that the curve has two components. The left one is obtained for $u_{1} \in[0.07650139 ; 0.27046582]$ and the right one for $u_{1} \in[-3.17251656 ;-2.61929914]$. Note that the borders of the two intervals are the roots of $w$, which can be computed explicitly, but, in order to avoid too long expressions, we displayed them numerically.

Based on the parametrization given in Equation (16), one can easily calculate (cf. [11] (Equation (6.8))) the curve of regression of the fixed axode, which is also displayed in Figure 10. 


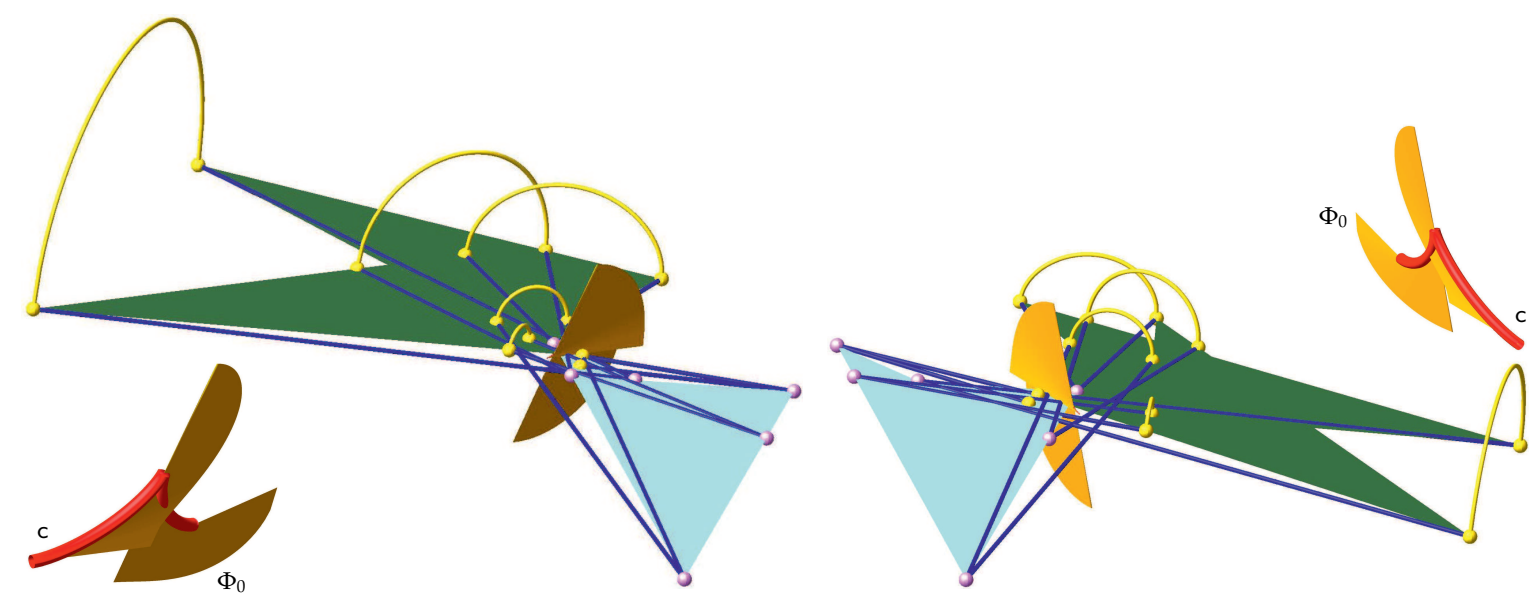

Figure 10. Trajectories of the platform points during the plane-symmetric self-motion between the flat poses 1 and 2 (left) and the flat poses 3 and 4 (right). Moreover, the fixed axodes $\Phi_{0}$ are displayed, which look like cones upon the first viewing. However, a blow up (in the lower left corner and upper right corner, respectively) of the region of the supposed vertex shows the line of regression c of $\Phi_{0}$. For the illustrated self-motion, the tangents of $c$ in the two end points span the carrier plane (xy-plane) of the flat poses. If one considers the complete self-motion, then c has four cusps (obtained by reflecting the illustrated curve at the $x y$-plane).

\subsection{Point-Symmetric Self-Motions of the Duporcq Hexapod}

Finally, we want to correct a statement given in [39] (Remark 4), where it is stated that

1. the Duporcq manipulator also has pure translational one-dimensional self-motions,

2. each two-dimensional line-symmetric self-motion of a Duporcq manipulator contains a pure translational one-dimensional sub-self-motion.

The first statement is true in contrast to the second one. In fact, the pure translational self-motion (which can be considered as point-symmetric self-motion) has two branching singularities, where they can switch into a 2-parametric line-symmetric self-motion. This can easily be seen as follows:

For the coordinatisation of the platform points and base points used in Section 3.4, the 1-parametric point-symmetric motion fulfills $e_{0}=e_{1}=e_{2}=f_{3}=0$. It can be computed by expressing $f_{1}$ from $\Lambda_{2}-\Lambda_{1}$ (which is linear in $f_{1}$ ). Plugging the resulting expression into $\Lambda_{1}$ implies a homogenous quadratic equation in $e_{3}, f_{0}, f_{2}$, which already represents the point-symmetric self-motion. By the additional condition $f_{0}=0$, we obtain the two mentioned branching singularities, which are again totally flat configurations of the Duporcq hexapod ( cf. Table 2 and Figure 11).

Table 2. The Study parameters of the two flat transition poses illustrated in Figure 11. As they result as roots of a polynomial of degree 2 , they can be computed explicitly, but in order to avoid too long expressions they are again displayed numerically.

\begin{tabular}{cccc}
\hline Flat Pose & $e_{3}$ & $f_{1}$ & $f_{2}$ \\
\hline 1 & 1 & 0.1406805116103807682 & -0.2234541534831142304 \\
2 & 1 & 2.9246864608666834522 & -1.0586559382600050357 \\
\hline
\end{tabular}



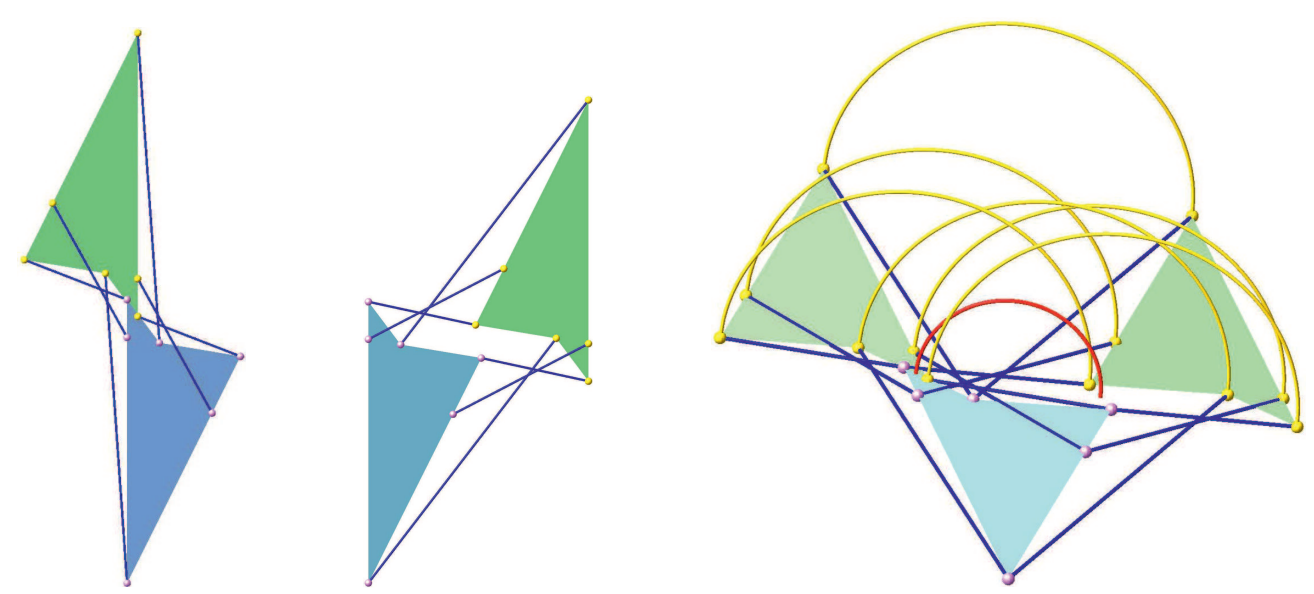

Figure 11. The first and second flat pose (left and center, respectively) and the translational self-motion between them (right). This circular translation can also be seen as a point-symmetric motion, where the corresponding curve (half-circle) is illustrated in red.

Finally it should be noted that there is no branching singularity between plane-symmetric self-motions and point-symmetric self-motions as $e_{0}=e_{1}=e_{2}=e_{3}=0$ has to hold, which contradicts the normalizing condition $N=1$. Summed up one can say, that the Duporcq hexapod is a twofold kinematotropic mechanism, as there are branching singularities between the two-dimensional line-symmetric self-motion and the one-dimensional

- point-symmetric self-motion,

- plane-symmetric self-motion.

Due to its kinematotropic behavior and its total flat branching singularities the Duporcq manipulator is possibly of interest for the design of deployable structures.

\section{Conclusions}

This paper gives a complete classification of hexapods with plane-symmetric self-motions. It turns out that besides the planar/spherical symmetric rollings with circular paths and two trivial cases (butterfly self-motion and two-dimensional spherical self-motion), only one further solution exists, which is the so-called Duporcq hexapod. This is the only manipulator possessing plane-symmetric self-motions, which are neither planar nor spherical motions (and therefore also no line-symmetric motions). Moreover, the Duporcq hexapod is may be of interest for the design of deployable structures due to its kinematotropic behavior and total flat branching singularities.

Funding: The author is supported by Grant No. P 24927-N25 of the Austrian Science Fund FWF within the project "Stewart Gough platforms with self-motions".

Conflicts of Interest: The author declares no conflict of interest.

\section{References}

1. Quetelet, L.A.J. Memoire sur une nouvelle maniere de considerer les caustiques, produites soit par reflexion soit par refraction. Brux. Nouv. Mem. 1826, 3, 89-140. (In French)

2. Tölke, J. Ebene euklidische und sphärische symmetrische Rollungen. Mech. Mach. Theory 1978, 13, 187-198. (In German) [CrossRef]

3. Bereis, R. Über die symmetrische Rollung. Österr. Ing. Arch. 1953, 7, 243-246. (In German)

4. Bottema, O. Characteristic properties of the symmetric plane motion. Proc. K. Ned. Akad. Wet. B 1972, 75, 145-151.

5. Darboux, G. Lecons sur la Theorie Generale des Surfaces; Gauthier-Villars: Paris, France, 1887. (In French) 
6. Krames, J. Über Fußpunktkurven von Regelflächen und eine besondere Klasse von Raumbewegungen (Über symmetrische Schrotungen I). Monatsh. Math. Phys. 1937, 45, 394-406. (In German) [CrossRef]

7. Selig, J.M.; Husty, M. Half-turns and line symmetric motions. Mech. Mach. Theory 2011, 46, $156-167$. [CrossRef]

8. Bottema, O.; Roth, B. Theoretical Kinematics; North-Holland: Amsterdam, The Netherlands, 1979.

9. Gallet, M.; Nawratil, G.; Schicho, J.; Selig, J.M. Mobile Icosapods. Adv. Appl. Math. 2017, 88, 1-25. [CrossRef]

10. Hernandez-Gutierrez, I. Screw Surfaces in the Analysis and Synthesis of Mechanisms. Ph.D. Thesis, Stanford University, Stanford, CA, USA, 1989.

11. Pottmann, H.; Wallner, J. Computational Line Geometry; Springer: Berlin/Heidelberg, Germany, 2001.

12. Kunze, S.; Stachel, H. Über ein sechsgliedriges räumliches Getriebe. Elem. Math. 1974, 29, 25-32. (In German)

13. Baker, J.E. The single screw reciprocal to the general plane-symmetric six-screw linkage. J. Geom. Graph. 1997, 1, 5-12.

14. Husty, M.E. Borel's and R. Bricard's Papers on Displacements with Spherical Paths and their Relevance to Self-Motions of Parallel Manipulators. In Proceedings of the International Symposium on History of Machines and Mechanisms-HMM 2000; Ceccarelli, M., Ed.; Kluwer: Dordrecht, The Netherlands, 2000; pp. 163-171.

15. Nawratil, G. Correcting Duporcq's theorem. Mech. Mach. Theory 2014, 73, 282-295. [CrossRef] [PubMed]

16. Karger, A. Singularities and self-motions of equiform platforms. Mech. Mach. Theory 2001, 36, 801-815. [CrossRef]

17. Karger, A. Singularities and self-motions of a special type of platforms. In Advances in Robot Kinematics: Theory and Applications; Lenarcic, J., Thomas, F., Eds.; Kluwer: Dordrecht, The Netherlands, 2002; pp. 155-164.

18. Karger, A. Parallel manipulators with simple geometrical structure. In Proceedings of the EUCOMES 08: The Second European Conference on Mechanism Science; Ceccarelli, M., Ed.; Springer: Dordrecht, The Netherlands, 2008; pp. 463-470.

19. Nawratil, G. Non-existence of planar projective Stewart Gough platforms with elliptic self-motions. In Computational Kinematics: Proceedings of the 6th International Workshop on Computational Kinematics (CK2013); Thomas, F., Perez Garcia, A., Eds.; Springer: Dordrecht, The Netherlands, 2013; pp. 49-57.

20. Nawratil, G. On equiform Stewart Gough platforms with self-motions. J. Geom. Graph. 2013, 17, $163-175$.

21. Nawratil, G. Congruent Stewart Gough platforms with non-translational self-motions. In Proceedings of the 16th International Conference on Geometry and Graphics; Schröcker, H.-P., Husty, M., Eds.; Innsbruck University Press: Innsbruck, Austria, 2014; pp. 204-215.

22. Nawratil, G. On the Self-Mobility of Point-Symmetric Hexapods. Symmetry 2014, 6, 954-974. [CrossRef]

23. Dietmaier, P. Forward kinematics and mobility criteria of one type of symmetric Stewart-Gough platforms. In Recent Advances in Robot Kinematics; Lenarcic, J., Parenti-Castelli, V., Eds.; Kluwer: Dordrecht, The Netherlands, 1996; pp. 379-388.

24. Karger, A.; Husty, M. Classification of all self-motions of the original Stewart-Gough platform. Comput. Aided Des. 1998, 30, 205-215. [CrossRef]

25. Nawratil, G. Self-motions of parallel manipulators associated with flexible octahedra. In Proceedings of the Austrian Robotics Workshop; Hofbaur, M., Husty, M., Eds.; Umit Lecture Notes: Hall in Tyrol, Austria, 2011; pp. 232-248.

26. Nawratil, G. Planar Stewart Gough platforms with a type II DM self-motion. J. Geom. 2011, 102, 149-169. [CrossRef]

27. Husty, M.L.; Karger, A. Self motions of Stewart-Gough platforms, an overview. In Proceedings of the Workshop on Fundamental Issues and Future Research Directions for Parallel Mechanisms and Manipulators, Quebec City, QC, Canada, 2-3 October 2002; Gosselin, C.M., Ebert-Uphoff, I., Eds.; pp. 131-141.

28. Nawratil, G. Introducing the theory of bonds for Stewart Gough platforms with self-motions. ASME J. Mech. Rob. 2014, 6. [CrossRef]

29. Gallet, M.; Nawratil, G.; Schicho, J. Liaison Linkages. J. Symb. Comput. 2017, 79, 65-98. [CrossRef]

30. Borel, E. Mémoire sur les déplacements à trajectoires sphériques. In Mém. Présent. Var. Sci. Acad. Sci. Natl. Inst. Fr. TOME XXXIII; Imprimerie Nationale: Paris, France, 1908; pp. 1-128. (In French)

31. Bricard, R. Mémoire sur les déplacements à trajectoires sphériques. J. École Polytech. 1906, 11, 1-96. (In French)

32. Karger, A. New Self-Motions of Parallel Manipulators. In Advances in Robot Kinematics: Analysis and Design; Lenarcic, J., Wenger, P., Eds.; Springer: Dordrecht, The Netherlands, 2008; pp. 275-282.

33. Karger, A. Self-motions of Stewart-Gough platforms. Comput. Aided Geom. Des. 2008, 25, 775-783. [CrossRef] 
34. Figliolini, G.; Angeles, J. The Spherical Equivalent of Bresse's Circles: The Case of Crossed Double-Crank Linkages. ASME J. Mech. Rob. 2014, 9. [CrossRef]

35. Glaeser, G.; Stachel, H.; Odehnal, B. The Universe of Conics; Springer Spektrum: Berlin/Heidelberg, Germany, 2016.

36. Husty, M.L. An algorithm for solving the direct kinematics of general Stewart-Gough platforms. Mech. Mach. Theory 1996, 31, 365-380. [CrossRef]

37. Borras, J.; Thomas, F.; Torras, C. On Delta Transforms. IEEE Trans. Robot. Autom. 2009, 25, 1225-1236. [CrossRef]

38. Duporcq, E. Sur un remarquable déplacement à deux paramétres. Bull. Soc. Math. Fr. 1901, 29, 1-4. (In French)

39. Nawratil, G.; Schicho, J. Duporcq Pentapods. ASME J. Mech. Rob. 2017, 9. [CrossRef]

40. Gogu, G. Branching singularities in kinematotropic parallel mechanisms. In Computational Kinematics: Proceedings of the 5th International Workshop on Computational Kinematics; Kecskemethy, A., Müller, A., Eds.; Springer: Berlin/Heidelberg, Germany, 2009; pp. 341-348.

41. Stachel, H. Flexible Polyhedral Surfaces With Two Flat Poses. Symmetry 2015, 7, 774-787. [CrossRef]

42. Wohlhart, K. Kinematotropic linkages. In Recent Advances in Robot Kinematics; Lenarcic, J., Parenti-Castelli, V., Eds.; Kluwer: Dordrecht, The Netherlands, 1996; pp. 359-368.

(C) 2018 by the authors. Licensee MDPI, Basel, Switzerland. This article is an open access article distributed under the terms and conditions of the Creative Commons Attribution (CC BY) license (http://creativecommons.org/licenses/by/4.0/). 